\title{
Three-dimensional, three-component flow field measurements with a single camera
}

\author{
Cierpka, Christian; Rossi, Massimiliano; Segura, Rodrigo; Kähler, Christian J. \\ Institut für Strömungsmechanik und Aerodynamik, Universität der Bundeswehr München \\ Werner-Heisenberg-Weg 39, 85577 Neubiberg
}

\section{Introduction:}

In many flow devices complex three-dimensional velocity fields are present and need to be characterized for further optimization. In order to perform such characterization, optical particle based techniques are a very advanced and reliable tool in fluid dynamics. For instance, 2D2C PIV (particle image velocimetry) can give $2 \mathrm{D}$ velocity information, with high spatial resolution, on the light sheet plane. Furthermore, extensions of this principle are used to measure the third velocity component. A stereoscopic setup, utilizing two cameras separated by a certain observation angle can be used. On the other hand, tomographic PIV takes advantage of four cameras. In this case, particle positions are reconstructed by a complex mathematical procedure. The velocity field is later determined by the $3 \mathrm{D}$ cross correlation of the reconstructed particle volumes at different times. The major difficulty for multi camera techniques, however, is the need for a very precise calibration and optical setup. Small errors in mapping the camera images to the position in real space can lead to large measurement uncertainties. Another problem arises from the fact that in many flows, e.g., in micromechanical devices, engines or other internal flows, the optical access is limited and multi camera techniques cannot be applied. Therefore, a measurement technique that uses a single optical access (and thus a single camera) and is able to determine the three-dimensional velocity field in the volume with high accuracy would be desirable.

\section{Measurement principle:}

To overcome the problems of multi camera approaches, a 3D PTV (particle tracking velocimetry) technique, based on astigmatic image aberrations was developed at the Bundeswehr Universität München. Using this new technique, it is possible to measure the complete three-dimensional velocity field in a volume with high accuracy using a single camera and thus a single optical access. The measurement principle is schematically shown in Fig. 1. Tracer particles that should ideally represent the fluid's velocity are seeded in the flow. The main idea is to code the depth position of these small tracer particles by astigmatic image aberrations [1]. This is accomplished by introducing a cylindrical lens into the observation optics as presented in Fig. 1. The axis of the cylindrical lens is aligned with the $x$-axis in the figure. In the $x z$-plane the cylindrical lens acts, in a first order approximation, as a flat window. Rotating the setup by $90^{\circ}$ (lower part of Fig. 1), it is clearly visible that the curvature of the cylindrical lens in the yz-plane causes the focal plane $F_{y z}$ to move closer to the objective than $F_{x z}$. Particles that are close to one focal plane, e.g., the $x$-axis focal plane $\left(F_{x z}\right)$, will give a small and sharp image in that axis. At the same time they are farther away from the infocus plane in the $y$-direction $\left(F_{y z}\right)$, which results in defocused, larger images in the $y$-axis. Thus, an elliptical image is formed on the CCD chip, with a small horizontal axis, denoted as $a_{x}$, and a large vertical axis, denoted as $a_{y}$. Conversely, when a particle is closer to the other focal plane, $a_{y}$ becomes smaller than $a_{x}$.

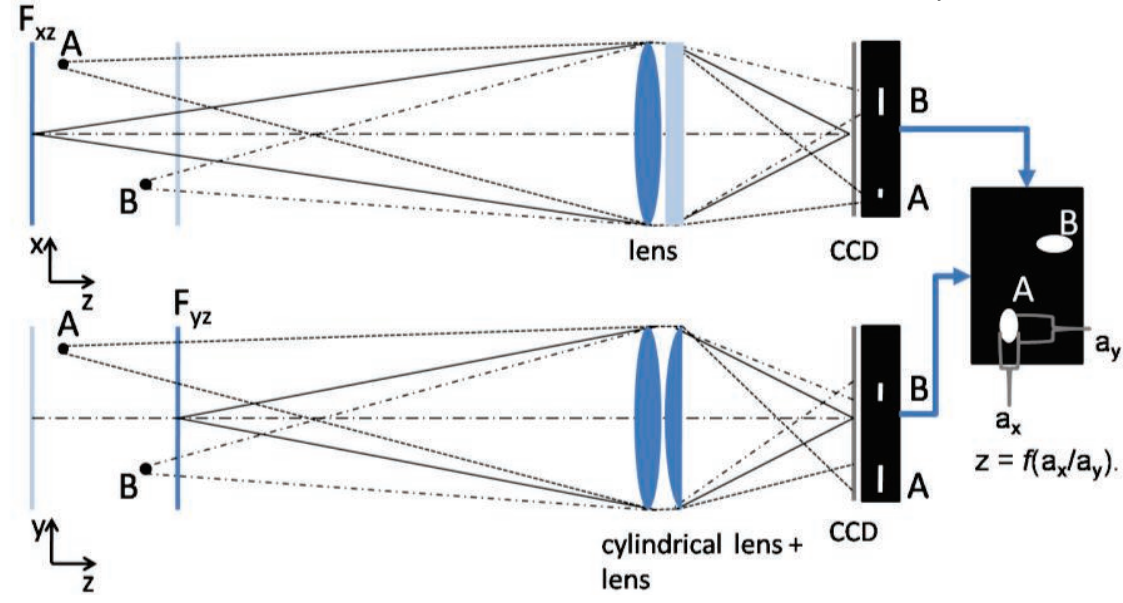

1. Ray tracing schematic of the optical set up for the astigmatism PTV. In the yz-plane the additional cylindrical lens 
shortens the distance to the in-focus plane $F_{x z}$ and the particle images appear deformed [1].

The position in the $x y$-plane is determined by a wavelet based procedure, which gives reliable results with subpixel accuracy up to high background noise levels ( 20\%). The out-of-plane or z-position can be estimated, knowing the size of the axes $a_{x}$ and $a_{y}$. Using an autocorrelation based algorithm with subpixel accuracy, $a_{x}$ and $a_{y}$ can be determined with high precission for particle images with $a_{x}, a_{y}>3$ pixel. To make the application of the technique as easy as possible, an intrinsic calibration procedure, based on the particle images themselves, $a_{x}$ and $a_{y}$, was developed. Since the defocus function of particles is well known, a model function can be fitted to the actual data [2]. Only the position of the two focal planes in real space $F_{x z}$ and $F_{y z}$ is necessary to calibrate the whole system. Since this can be done outside of the device (microchannel, combustion chamber, internal engine flow), the system is directly applicable and insensitive to misalignments in the setup. A complete treatment of the calibration approach is beyond the scope of this paper. The interested reader is referred to [2].

The focal length of the cylindrical lens determines the depth of the measurement volume. In general, the larger the focal length is, the closer are both focal planes and the smaller is the measurement depth. The proper choice of the cylindrical lens enables the user to adjust the measurement volume according to the experimental needs.

If the 3D positions $\boldsymbol{x}_{1}$ and $\boldsymbol{x}_{2}$ are known in the volume at two different time instants $t_{1}$ and $t_{2}$, the 3D velocity vector can be estimated to be $\boldsymbol{u}=\left(\boldsymbol{x}_{2}-\boldsymbol{x}_{1}\right) /\left(t_{2}-t_{1}\right)$ by a nearest neighbor search.

\section{Applications:}

Using fast cameras, astigmatism PTV can be used to resolve particle trajectories in space and time. This helped, for example, to understand the generation of an electro kinetic microvortex and the trapping of particles as can be seen in Fig. 2. Such a vortex was generated by changing the electric properties of a fluid between two electrodes, by heating with an infrared laser beam [3]. Using ITO glass electrodes it is possible to generate such a vortex on any position on the substrate. The flow regime itself depends mainly on the frequency of the AC electric field and can be varied widely. The flow induced by the microvortex can be used to mix or move fluids or to manipulate particles. For example, the separation of larger particles from smaller ones is a common task in modern lab-on-a-chip applications.

The experiments, for which the results are shown in Fig. 3, were performed in collaboration with the Purdue University, USA. On the left hand side, selected 3D trajectories of an electrothermally driven microvortex are shown in the volume. The torroidal structure of this kind of vortex can be seen clearly.
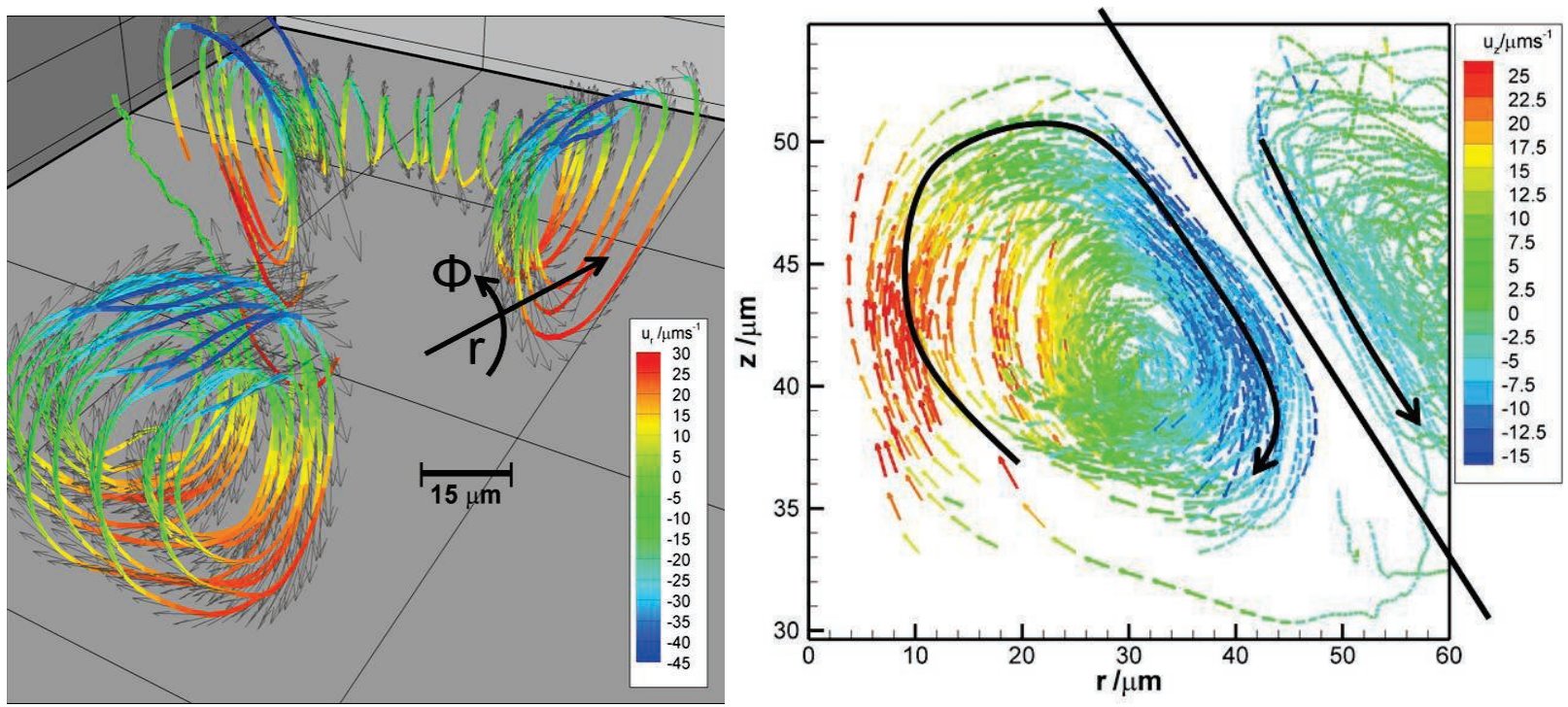

2. Three-dimensional particle trajectories and corresponding velocity vectors in the vortex center. Color represents the radial velocity component (left). 2D representation along the depth direction of the vortex. The boundary between primary and secondary vortex can be clearly seen (right). Color corresponds to the axial velocity [3].

Prior to the 3D3C measurements it was not clear if the vortex was axis symmetric. The measurement volume for this particular setup was $500 \times 500 \times 65 \mu^{3}$. Since the whole vortex could be measured without traversing, the inclination becomes evident in the results displayed on the right hand side of Fig. 2. A clear boundary was observed between a primary vortex, driven by the infrared laser, and the secondary vortex, which is 
generated due to continuity. Particles that are trapped in the primary vortex cannot leave it. At the lower part of the figure, one can actually see that particles from outside could be trapped in the primary vortex and will rotate about its center on a torroidal trajectory. A comparison with numerical results helped to validate the assumptions regarding the boundary conditions for the simulation.

The technique is not limited to microscopic flows, but can be used on every length scale. A very complex, fully three-dimensional and stationary flow evolves for example during the electrodeposition of copper under the influence of an external magnetic field [4]. The interplay of different volume forcers, such as the Lorentz force and the magnetic field gradient force, induce mixing which can enhance the deposition rate. This effect is commonly known as "MHD-effect". The understanding of these forces is hence crucial in the proper design of effective electrochemical systems. In Fig. 3 , an electrochemical cell $\left(10 \times 10 \times 80 \mathrm{~mm}^{3}\right)$ is shown. The bottom electrode was a thin, gold-covered glass disc with a thickness of $70 \mu \mathrm{m}$ that served as working electrode (WE). A reference electrode (RE) was connected to the cell close to the WE. The top electrode serves as counter electrode (CE) and was made of a Pt sheet. Since both electrodes have the same size, the electric field in the cell is uniform. Details of the setup, the applied electrical field and solutions can be found in [4]. The template beneath the cell with the iron wire produces a defined gradient in the magnetic field of the permanent magnet, thus giving rise to the magnetic field gradient force, which acts towards the edge of the Fe-wire. The influence on the deposition can be clearly seen in the right part of Fig. 3, where a dark ring proves enhanced copper deposition close to the wire.

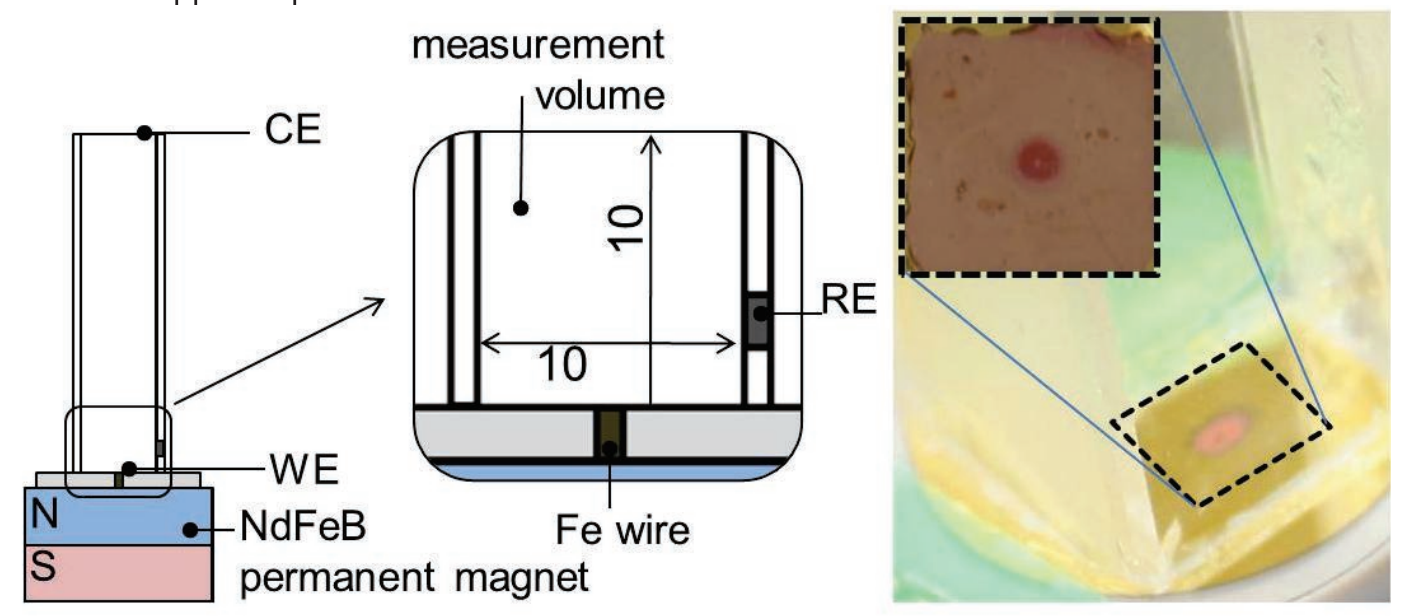

3. Setup of the electrochemical cell (left), with working electrode (WE), counter electrode (CE) and the iron wire template. Increased deposition of copper in regions of strong gradients (right) [4].
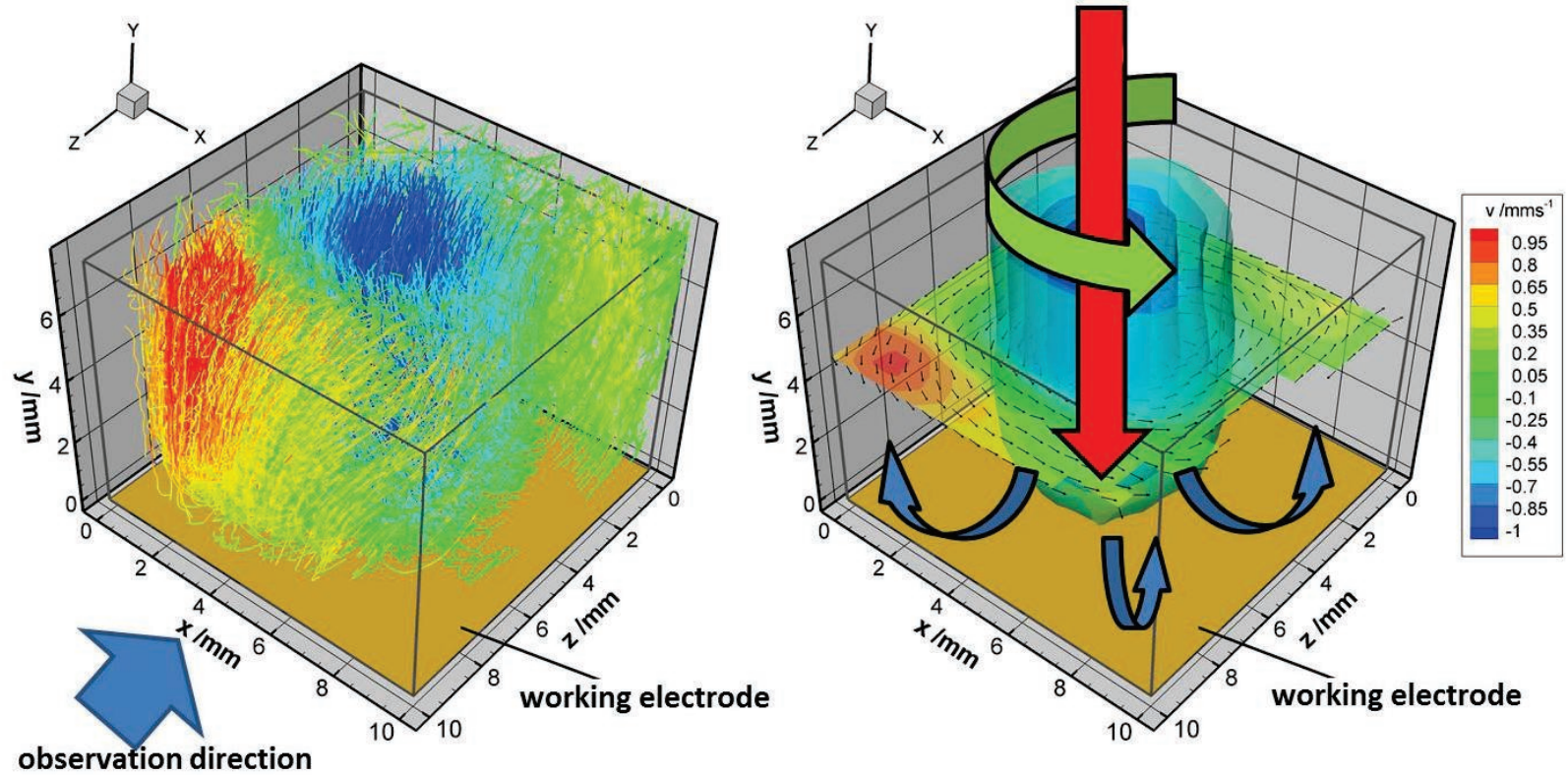

4. Three-dimensional particle trajectories (left) and iso-contours of the velocity towards the iron wire and indication of the rotational flow induced by the Lorentz force (green), the downstream flow induced by the magnetic gradient force (red) and the upward flow due to continuity (blue) (right). 
The experiments were performed in collaboration with the Leibniz Institute for Solid State and Materials Research in Dresden, Germany. A long range microscope, which basically works like a telescope, was used for the image acquisition. The working distance was larger than $0.5 \mathrm{~m}$ and the measured volume had a size of $10 \times 10 \times 10 \mathrm{~mm}^{3}$. Within this volume the flow was measured for about 12 minutes, allowing to reconstruct thousands of particle trajectories, as can be seen in Fig. 4 on the left side. On the right side of the same figure, iso-contours of the complex velocity field are shown. The Lorentz force, induces a rotational flow, that is highlighted by the velocity vectors in the slice and is indicated by the green arrow. If the Lorentz force would be only force present, the well known tea-cup flow, where the tea leaves would meet in the center would be established and, in the center, an upward flow would be visible. However, the influence of the magnetic gradient force causes a jet like flow towards the working electrode. This flow can be clearly seen by the blue iso-surfaces of the velocity in the $z$-direction and is indicated with a red arrow. However, due to continuity, the fluid has to flow upwards in the corners of the cell, as indicated in blue. The influence of the magnetic field gradient force was never experimentally measured before. 3D3C time resolved measurements were needed to describe its influence in detail and to understand the underlying physics.

Since the particle positions in the volume are known, the volumetric particle distributions can be used to reconstruct fluid interfaces, e.g., to understand particle agglomeration at walls or mixing processes [5]. Due to the low Reynolds numbers in microfluidic devices, the flow is very often laminar. Hence, mixing due to turbulence is not possible and most mass transfer phenomena are diffusion based. An increase of the interface between two reagents, for example, would increase the mass transfer by diffusion via this interface and is desirable. In Fig. 4 the reconstructed interface between two fluids can be seen. The experiment was performed in collaboration with the Politecnico di Torino, Italy. A passive micromixer with a cross section of $200 \times 70 \mu \mathrm{m}^{2}$ was used. This mixer consists of two flow inlets and an outlet. Downstream of the junction that connects the two inlets the channel is curved. With increasing velocity, inertial effects promote the formation of a secondary flow. This process is characterized by the Dean number, which is proportional to the Reynolds number. For the characterization of the micromixer, one inlet (1) was fed with a particle seeded fluid (distilled water and latex microspheres), while the other inlet (2) carried clean distilled water. The position of the tracer particles therefore represents the region of fluid from inlet 1 and is highlighted in red in Fig. 5. A numerical diffusion scheme was used to reconstruct the interface between the two fluids. The effect of the secondary flow is clearly visible. For the lower Reynolds number of $\operatorname{Re}=20$, there is almost no deformation of the interface and the fluid streams are nicely aligned as they would be in a straight channel. For the higher Reynolds number of $\mathrm{Re}=100$ the influence of the secondary flow due to the curvature can be clearly seen. The interface between both streams highly increases and thus the mixer works more effectively. Interestingly, since this flow is completely reversible, both streams are aligned again at the outlet.
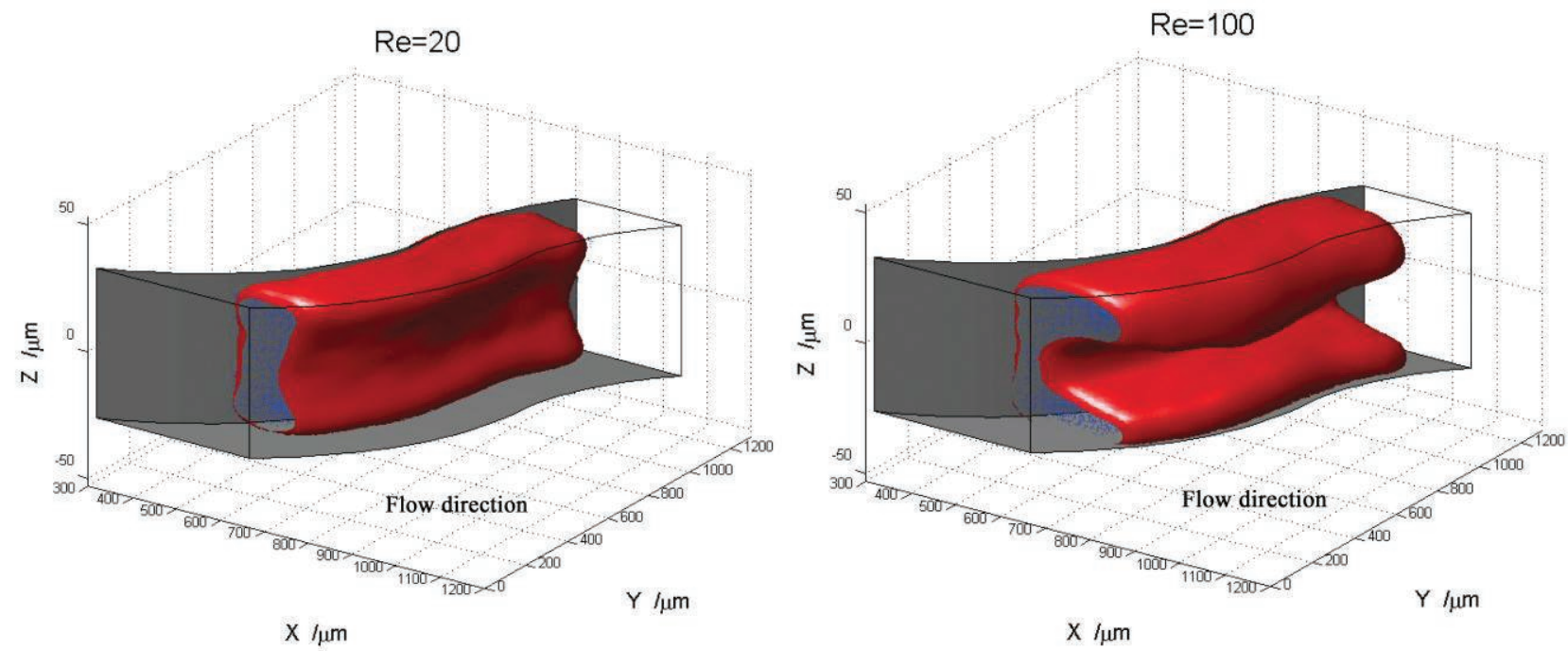

5. Reconstruction of the fluid interface in a micro mixer $\left(\operatorname{Re}_{\text {left }}<\operatorname{Re}_{\text {rigth }}\right)$. The Reynolds number effects on the interfaces between the particle laden flow on one side and the flow without particles in a micro mixer is clearly visible [5].

\section{Conclusion:}

The presented 3D3C astigmatism PTV method enables the user to measure the 3D velocity field within a volume with a single camera. Different applications show the great potential of the technique to be used in various multi physics problems. The technique itself is thus not limited to liquids. A preliminary study in air, using DEHS seeding particles showed promising results. A major advantage in comparison to multi camera 
techniques for flow measurments is that only one optical access is necessary. This makes the technique applicable to internal flows in complex geometries. Volume illumination can be applied and no special care for the light sheet generation has to be taken. Measurements in liquids were already successfully performed. Since just one camera is used, no mapping errors between different cameras will decrease the accuracy of the method.

Nearly every existing 2D2C PIV system can be upgraded to measure the 3D3C velocity field, only by including a cheap cylindrical lens. These lenses can be bought from the shelf, without the need for additional expensive equipment like cameras. The measurement depth can be adjusted, changing the focal length of the cylindrical lens. Due to the intrinsic calibration, the system is able to handle unwanted image aberrations and can be used without expert knowledge.

\section{Acknowledgements:}

Financial support from the German Research Foundation (DFG) is gratefully acknowledged.

\section{References:}

1. C. Cierpka, R. Segura, R. Hain, C.J. Kähler: A simple single camera 3C3D velocity measurement technique without errors due to depth of correlation and spatial averaging for microfluidics. Measurements, Science and Technology 21, 2010, 045401, DOI: 10.1088/0957-0233/21/4/045401

2. C. Cierpka, M. Rossi, R. Segura, C.J. Kähler: On the calibration of astigmatism particle tracking velocimetry for

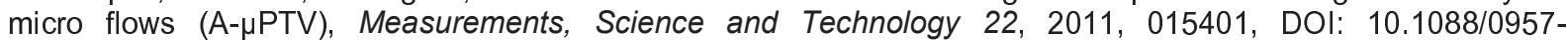
0233/22/1/015401

3. A. Kumar, C. Cierpka, S.J. Williams, C.J. Kähler, S.T. Wereley: 3D3C velocimetry measurements of an electrothermal microvortex using wavefront deformation PTV and a single camera. Microfluidics and Nanofluidics, 2010, DOI: 10.1007/s10404-010-0674-4

4. C. Cierpka, K. Tschulik, R. Segura, M. Uhlemann, C.J. Kähler: Zeitaufgelöste 3D3C Geschwindigkeitsfeldmessungen mit der fernmikroskopischen Astigmatismus PTV zur Analyse der elektrochemischen Kupferabscheidung, Technisches Messen, zum Druck angenommen

5. F. Mastrangelo, M. Rossi, C. Cierpka, R. Segura, C.J. Kähler: Reconstruction of the interface between two fluids in microfluidic-mixers using astigmatic particle imaging, $2^{\text {nd }}$ European Conference in Microfluidics, 08.-10.12.2010, Toulouse, France 